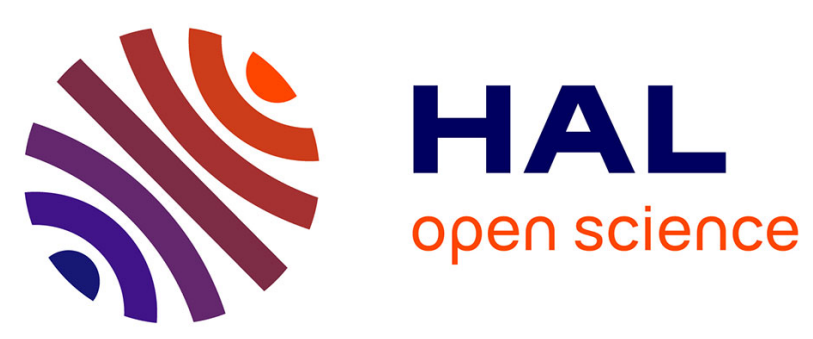

\title{
Multiscale Modeling of Dust Charging in Simulated Lunar Environment Conditions
}

Pauline Oudayer, Loanne Monnin, Jean-Charles Matéo-Vélez, Sébastien Hess, Pierre Sarrailh, Gaël Murat, Jean-François Roussel

\section{- To cite this version:}

Pauline Oudayer, Loanne Monnin, Jean-Charles Matéo-Vélez, Sébastien Hess, Pierre Sarrailh, et al.. Multiscale Modeling of Dust Charging in Simulated Lunar Environment Conditions. IEEE Transactions on Plasma Science, 2019, 47 (8), pp.3710-3716. 10.1109/TPS.2019.2919932 . hal-02315282

\author{
HAL Id: hal-02315282 \\ https://hal.science/hal-02315282
}

Submitted on 14 Oct 2019

HAL is a multi-disciplinary open access archive for the deposit and dissemination of scientific research documents, whether they are published or not. The documents may come from teaching and research institutions in France or abroad, or from public or private research centers.
L'archive ouverte pluridisciplinaire HAL, est destinée au dépôt et à la diffusion de documents scientifiques de niveau recherche, publiés ou non, émanant des établissements d'enseignement et de recherche français ou étrangers, des laboratoires publics ou privés. 


\title{
Multiscale Modelling of Dust Charging in Simulated Lunar Environment Conditions
}

\author{
P. Oudayer ${ }^{(1)}$, L. Monnin ${ }^{(1)}$, J.-C. Matéo-Vélez ${ }^{(1)}$, S. L. G. Hess ${ }^{(1)}$, P. Sarrailh ${ }^{(1)}$, G. Murat ${ }^{(1)}$, J.-F. Roussel ${ }^{(1)}$ \\ ${ }^{(1)}$ ONERA - The French Aerospace Lab, Toulouse, France
}

\begin{abstract}
A key aspect of dust adhesion to space equipment is the accumulation of charge under the space plasma environment. Recent models and experiments show possible negative charging of dust grains under VUV illumination. Macroscopic potential measurements conducted during a test campaign show that both positive and negative average charging can be reached under VUV irradiation pending on vacuum chamber configuration, suggesting that both situations can exist at lunar surface. Simulations of dust charging at microscopic scale are conducted with the SPIS software to evaluate electrical charge and electric field amplifications induced by the granular structure of the lunar regolith. A multi-layer pile of dust is modelled under lunar conditions. Grains from the first two layers tend to microscopically acquire both negative and positive charge patches when illuminated with a $45^{\circ} \mathrm{VUV}$ incidence angle, this differential charging being less pronounced under normal incidence angle. It is also found that dust deeply buried in the lunar soil may charge more negative due to the collection of environmental electron only. This effect is thought to reinforce the grain supercharging model presented by other authors. We show however that such charge development may be limited by electrical conduction pending on dust electrical properties.
\end{abstract}

Index Terms-dust lofting, lunar dust, electrical charge.

\section{INTRODUCTION}

One of the main fallouts from the Apollo program is the problem of lunar dust adhesion which was initially assumed to be of secondary importance. The dust layer covering the surface of the Moon tended to stick to any human-made equipment, and therefore complicated the full exploitation of the facilities. It also led to human body undesired effects such as eye irritation, respiratory problems, hay fever symptoms, etc [1]. The next space missions will be initiated by robots, with no human intervention and no longer confined to the Moon: planets, comets and asteroids are also targeted. It therefore becomes more and more crucial to anticipate and bring under control the overall dust adhesion problem. In order to achieve this goal, the first priority is to understand the physical phenomena at stake throughout experiments. Particle 2019).

This work was conducted thanks to an ONERA Ph.D. fellowship (2016-

P. Oudayer, L. Monnin, J.-C. Matéo-Vélez, S. Hess, P. Sarrailh, G. Murat and J.-F. Roussel are with ONERA/DPHY, Université de Toulouse, F-31055 Toulouse, France (email: mateo@onera.fr). collection on equipment surfaces may be due to human and robotic activity, leading to dust mobilization from the object soil. Particle levitation from the Moon surface may also be due to the space environment interaction with its surface. Indeed, the Moon surface is likely to be negatively charged due to the solar-wind electron collection. On the day-side however, photoemission phenomenon occurs and leads the surface to be positively charged. At the frontier of day and night-side (called terminator), the presence of both negative and positive charges on small distances create an electric field believed to be strong enough to electrostatically loft dust particle at the Moon surface during the so-called horizon glow phenomenon ([2]-[3]). This phenomenon is mainly seen at dawn and dusk. Both experimental and numerical investigations have recently been conducted to model the effect of the lunar plasma environment on dust charging and lofting ([4], [5], [6]).

Independently of the origin of dust deposit onto surfaces, i.e. mobilization by robotic/rover/lander/human activity or natural lofting induced by the environment, adhesion of dust to exploration unit surfaces is mainly due to two forces: the Van der Waals force, which is a distance-dependent cohesive force, and the electrostatic forces [7] which are a direct result of the lunar space environment since the charge depends on the complex interactions between the ambient plasma and dust. The intensity of these forces remains poorly quantified within the regolith.

In day-side and under VUV irradiation, the dust layer is expected to be positively charged [8]. Experimental evidence of positively charged dust mobilization induced by VUV exposure was shown by [5]. Their setup consisted in applying a horizontal electric field using a positive electrode and a negative electrode both located a few millimeters from a dust layer. Dusts were collected by the negatively biased electrode and not on by a positively biased. In other experimental configurations however, with a grid located above the dust layer, dust mobilization occurred after VUV exposure only when a positive voltage was applied to the grid (Prof. M. Horanyi, private communication). This suggests that different experimental setups can produce different charging at large scale, i.e. at a few millimeters to centimeters scale, what is called macroscopic scale in this paper. One possible explanation for this is the presence of macroscopic electric fields that deflect the electrons emitted by VUV impacts on illuminated surfaces. In addition, complex microscopic charging patterns are supposed to be generated due to the interstices between dust grains that make charged particle 
collection and emission very different from a smooth flat surface [4]. Due to the very loose packing at the surface of the Moon, photoemission phenomena can occur on dust buried just beneath the top dust layer, i.e. what can be called the second dust layer. Some of the emitted photoelectrons can be collected at the bottom of particles of the first layer, locally creating a negative potential spot, while the upper side of the layer remains positively charged due to photoemission. This model was confirmed by numerical simulations [9]. Complementary experimental investigations showed that a typical difference potential of $-2.5 \mathrm{~V}$ can be achieved between illuminated side and shaded side of two dust grains [4].

To our knowledge no attempts have been done to validate the numerical modelling of dust charging with dedicated experiments both at the microscopic scale of the dust grains and at the macroscopic scale of the experimental setup. Both scales are indeed involved in the collection of charge carriers (electrons most of the time) by dust. The objective of this paper is to propose a combined study of dust layer charging under VUV exposure both experimentally and numerically.

Section II of this paper is dedicated to simulations of a dust pile under plasma environment using a microscopic grain arrangement similar to [9]. In Section III we replicate laboratory experiments with simulation at both macroscopic and microscopic scales. Main results are discussed and concluded in Section IV.

\section{MicROSCOPIC MODELLING OF DUST LAYERS UNDER SPACE ENVIRONMENT}

\section{A. Modelling}

Numerical simulations are conducted with the open-source SPIS software version 6.0 (www.spis.org). A superposition of three grains layers is simulated. The simulation domain is bounded by a rectangular domain of dimensions $0.8 \mathrm{~mm}, 0.6$ $\mathrm{mm}$ and $1.8 \mathrm{~mm}$ along the $\mathrm{X}, \mathrm{Y}$ and $\mathrm{Z}$ axis respectively. A potential of $0 \mathrm{~V}$ is applied on top of the simulation domain. The bottom boundary is set to a floating potential. Periodic conditions along the $\mathrm{X}$ and $\mathrm{Y}$ axis and reflective conditions along the $\mathrm{X}$ axis are implemented in order to simulate an infinite surface. Each dust grain is $100 \mu \mathrm{m}$ in radius and separated by $1 / 10^{\text {th }}$ of the particle size with respect to its neighbors. The grains are not in contact with each other. Dust layers lay onto a flat surface, simulating the rest of it. The SPIS material used to characterize the dust refers to "Lunar dust 71501 Mare" and a bulk conductivity of $10^{-14} \mathrm{~S} / \mathrm{m}$ is imposed, as well as a surface resistivity of $10^{15} \Omega$. No conduction path is modelled between each dust grain.

Two sets of simulations have been performed. The first one corresponds to a Sun incidence and to solar wind incidence angle $\alpha$ of $45^{\circ}$. The second one corresponds to a normal incidence. Solar wind plasma particles dynamics, i.e. electrons and protons, are simulated by Particle-In-Cell modeling. Maxwellian distributions are injected on the top boundary. Secondary electron emission by electron impacts (SEE) and by photoelectrons are simulated following PIC modelling and assuming Maxwellian distributions with a temperature of 2.2 $\mathrm{eV}$. Plasma parameters are gathered in Table I. The plasma parameters used during a previous study with $\alpha$ equal to $45^{\circ}$ are reported in Table II [9].

TABLE I

SIMULATION PARAMETERS (PRESENT WORK)

\begin{tabular}{lccc}
\hline \hline & Photoelectrons & Electrons & Ions \\
\hline Debye length $(\mathrm{m})$ & $\mathrm{x}$ & 7.4 & 7.4 \\
Drift Velocity $(\mathrm{m} / \mathrm{s})$ & $\mathrm{N} / \mathrm{A}$ & $4.3 \times 10^{5}$ & $4.3 \times 10^{5}$ \\
Thermal velocity $(\mathrm{m} / \mathrm{s})$ & $8.8 \times 10^{5}$ & $2 \times 10^{6}$ & $5 \times 10^{4}$ \\
Temperature $(\mathrm{eV})$ & 2.2 & 10 & 10 \\
Current density $\left(\mathrm{A} / \mathrm{m}^{2}\right)$ & $4.5 \times 10^{-6}$ & $8 \times 10^{-7}$ & $7 \times 10^{-7}$ \\
Density $\left(\# / \mathrm{m}^{3}\right)$ & $\mathrm{x}$ & $10^{7}$ & $10^{7}$ \\
& & & \\
\hline \hline
\end{tabular}

TABLE II

SIMULATION PARAMETERS (LITERATURE)

\begin{tabular}{lccc}
\multicolumn{3}{c}{ SIMULATION PARAMETERS (LITERATURE) } \\
\hline & Photoelectrons & Electrons & Ions \\
\hline $\begin{array}{l}\text { Debye length }(\mathrm{m}) \\
\begin{array}{l}\text { Characteristic velocity } \\
(\mathrm{m} / \mathrm{s})\end{array}\end{array}$ & 1 & 15 & 15 \\
$\begin{array}{l}\text { Characteristic energy } \\
(\mathrm{eV})\end{array}$ & 1 & $2 \times 10^{6}$ & $4 \times 10^{5}$ \\
$\begin{array}{l}\text { Current density }\left(\mathrm{A} / \mathrm{m}^{2}\right) \\
\text { Density }\left(\# / \mathrm{m}^{3}\right)\end{array}$ & $4 \times 10^{-6}$ & $1.5 \times 10^{-6}$ & $3 \times 10^{-7}$ \\
& $10^{8}$ & $5 \times 10^{6}$ & $5 \times 10^{6}$ \\
\hline \hline
\end{tabular}

\section{B. Results}

Fig. 1 presents the surface potential reached at equilibrium after about $200 \mathrm{~s}$ for $\alpha=45^{\circ}$ and neglecting SEE.

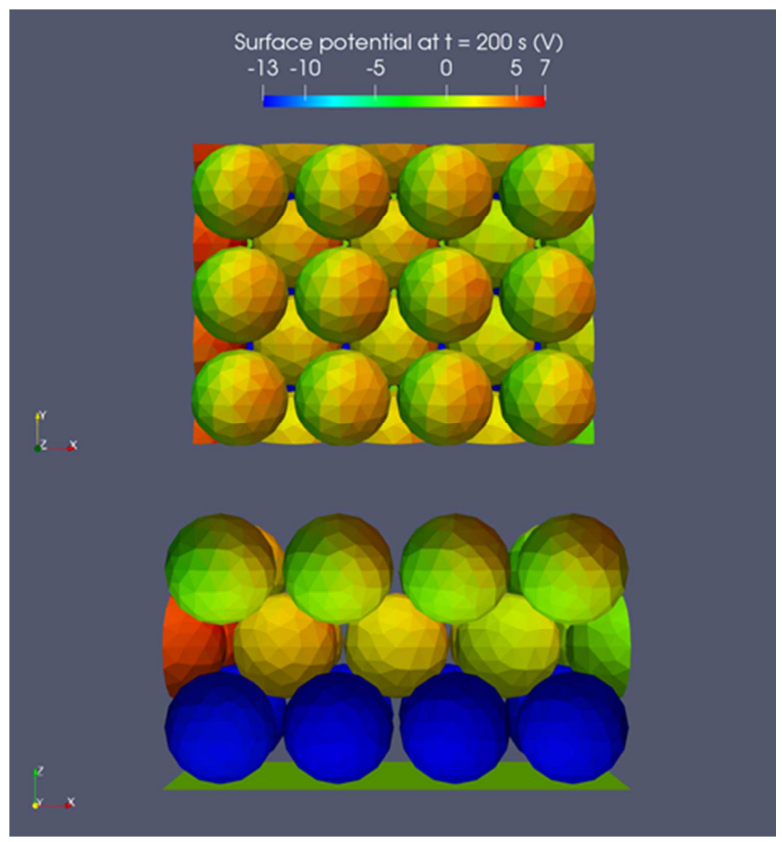

Fig. 1. Surface potential at equilibrium with $\alpha=45^{\circ}$ and without SEE. Bottom figure is the cross section along the Y-axis.

Simulations show the apparition of both positive and negative patches on top and at the bottom of the first layer, respectively. As in [9], photoemission on top of the first layer generates positive charge while photoemission on the second 
layer generates negative charges on the rear side of the top layer. The simulated differential potential obtained in this work is about $-7.5 \mathrm{~V}$ between the rear and front sides of the top layer. This is comparable to the $-2.5 \mathrm{~V}$ voltage obtained experimentally on macroscopic objects mimicking dust under VUV illumination [4]. However, the average potentials on the top layer and on the second layer are +1 and $+2.5 \mathrm{~V}$, respectively, suggesting super charging is compensated in average by photoemission on the first layer top surface. This equilibrium state is reached after about 10 seconds. The average potential reached after 200 seconds on the third layer is $-13 \mathrm{~V}$. This layer is not illuminated with directional VUV. It collects only solar wind electrons that find their way between grain interstices due to electrostatic deflection by the first and second layers. This collection needs a longer duration to reach equilibrium because electron fluxes are reduced by the presence of the first and second layers. The electric field obtained at 200 seconds is plotted in Fig. 2. Between, the top and second layer, we observe an electric field slightly greater than $2 \times 10^{5} \mathrm{~V} / \mathrm{m}$ which is in good agreement with previous results [9]. The electric field between the second and third layer is a bit less than one order of magnitude greater than between the first and the second. That suggests that the maximum grain super charging occurs quite deeply in the soil.

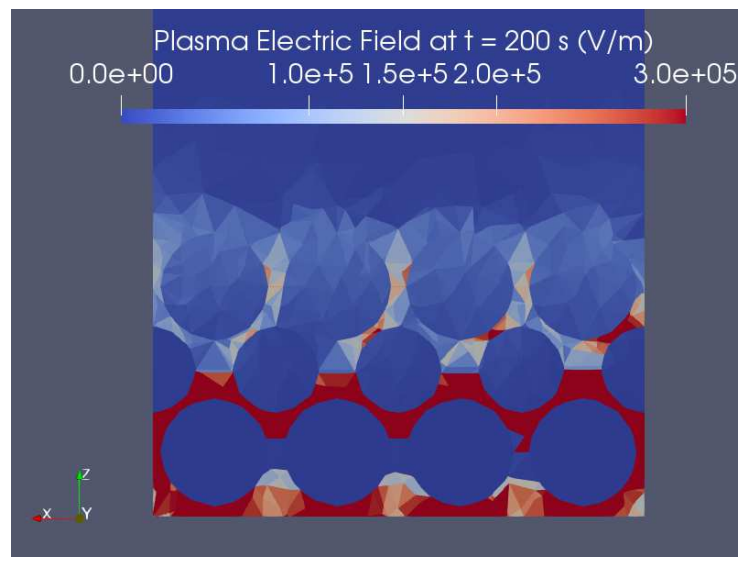

Fig. 2. Electric field at equilibrium in the same configuration as in Fig. 1.

Secondary electron emission impact is also studied. The main change when adding SEE is that the third layer is less negatively charged than previously, as seen on Fig. 3, i.e. -10 $\mathrm{V}$ instead of - $13 \mathrm{~V}$ after 200 seconds.

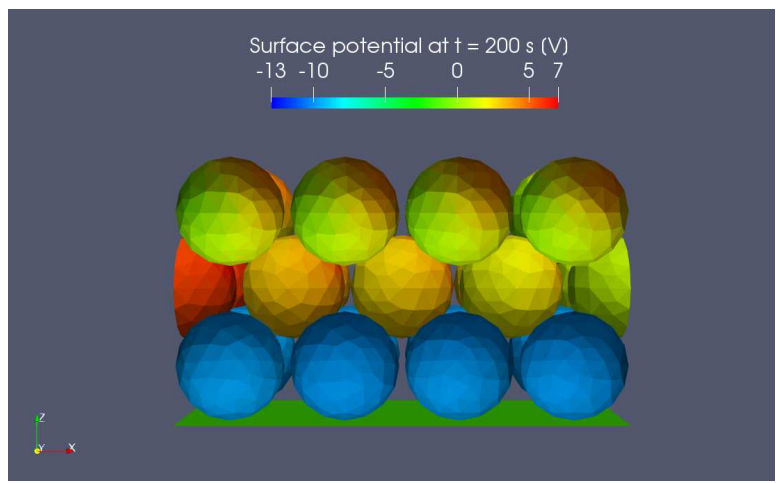

Fig. 3. Surface potential at equilibrium, with $\alpha=45^{\circ}$ and with SEE

Fig. 3 presents the surface potentials for $\alpha=45^{\circ}$ and with SEE. No significant change is observed on the top and second layer because their potential is controlled by the photoemission process rather SEE. The third layer is a bit less negative $(-10 \mathrm{~V})$ than in the previous case because SEE tends to limit the negative current balance. SEE is important to model mainly for the deeper layers thus.

Results obtained with $\alpha=0^{\circ}$ and with SEE are presented in Fig. 4. It appears that the negative and positive patches are less pronounced. The potential differences between the top layer front and rear sides reach about $-3.5 \mathrm{~V}$. This is quite well in agreement with the $-2.5 \mathrm{~V}$ measured in [4]. For the second layer, the charging behavior is comparable to the previous case.

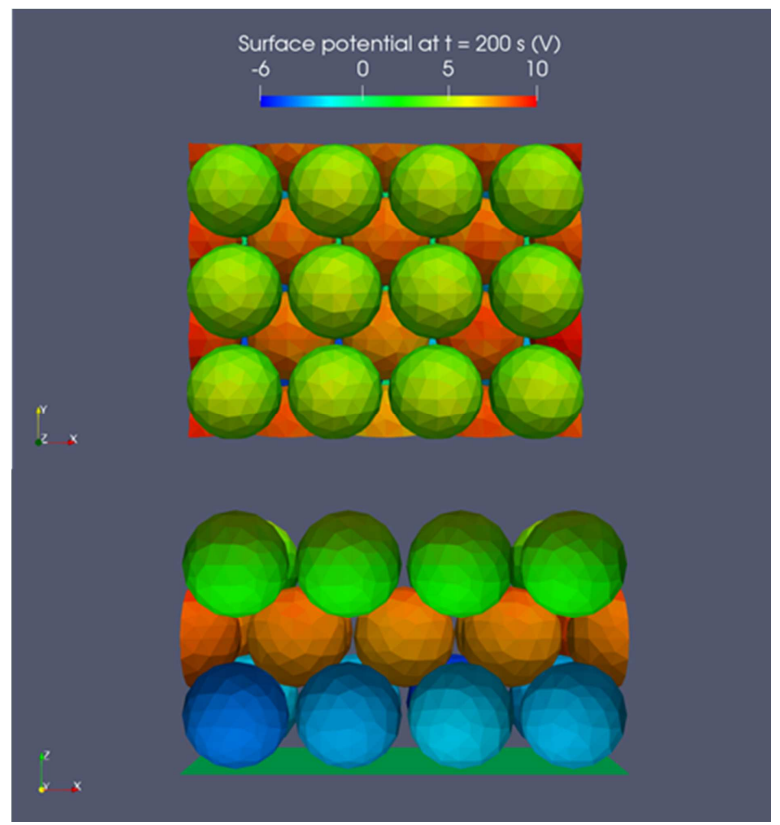

Fig. 4. Surface potential at equilibrium for $\alpha=0^{\circ}$ and with SEE. Bottom figure is the cross section along Y-axis.

The main difference between this work and the previous study reported in [9] is the number of grain layers. In [9], one layer and a half is simulated instead of three in the present 
work. Same observations are made for the top layers: apparition of positive charges where photoemission occurs (on top of the first and second layer) and negative charges where photoelectrons are collected (at the bottom of the first layer). The same qualitative behaviors are observed for changes in the solar wind electron density and temperature within a factor of 2. The theory of grain super charging seems thus to be corroborated by the present results [4]. In this work, we showed that grains buried beneath what we call the third layer can charge highly negative due to the infiltration of solar wind electron between the top layers. This may contribute to some change in the estimation of electrostatic forces.

\section{MULTISCALE MODELLING IN SIMULATED LUNAR ENVIRONMENT}

Microscopic scale modelling is thus possible to assess using numerical tools. Validating these tools with ground experiments is however difficult to achieve because of the difficulties to perform such experimental investigation at grain scale. In this section, we present experiments conducted to understand macroscopic charging and the efforts made to model these experiments with SPIS at this same macroscopic scale and at microscopic scale as well.

\section{A. Experimental setup}

Experiments have been conducted in the DROP vacuum chamber described in [5]. This facility is a cylindrical tank of dimension 40 centimeters in diameter and 40 centimeters in length covered with a hemispherical cap where a pressure of $10^{-6}$ mbar is obtained with a turbo-molecular pump. The chamber is equipped with a VUV deuterium lamp emitting photons from $120 \mathrm{~nm}$ to $400 \mathrm{~nm}$. The illuminated zone is about 10 centimeters in diameter, the lamp being placed 35 centimeters above the sample.

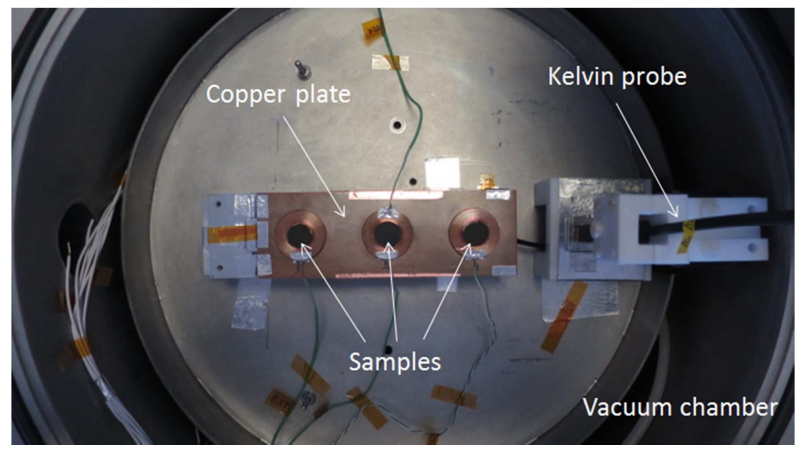

Fig. 5. Experimental setup inside the DROP chamber

Fig. 5 presents the setup used for surface potential measurements after VUV exposure. A total of three samples can be installed at the center of a holder plate made of copper. The samples are electrically connected to a bias voltage supply. A contactless Kelvin probe is mounted on a translating axis to measure the surface potential profile. The photoelectron flux emitted from a copper reference sample is about $40 \mathrm{nA} / \mathrm{cm}^{2}$ [10], which is about 20 times the expected photoemission flux PEY at 1 AU from the Sun. The UV lamp irradiance is thus supposed equivalent to 20 times the VUV flux at 1 AU from the Sun. Lunar dust simulant photoemission flux was an unknown parameter of the test setup and has to be determined.

Measurements of the potential are made using a Kelvin probe which is placed $2 \mathrm{~mm}$ above the copper plate. The sample thickness is about $200 \mu \mathrm{m}$, so the probe is placed 1.8 $\mathrm{mm}$ away from the dust layer. Samples used for the experiment are SMA_A lunar dust simulant, which grain size is inferior to $25 \mu \mathrm{m}$ [11]. The average grain size is $8 \mu \mathrm{m}$. The layer is made by depositing dust into a small container of 1.5 $\mathrm{cm}$ of diameter using a height gauge to obtain a thickness of about $200 \mu \mathrm{m}$. After pumping the vacuum chamber down to $10^{-6} \mathrm{mbar}$, the dust layer is irradiated for a duration of two minutes. After this period, the potential is measured above the centre point of the dust deposit. The first twenty seconds of data are lost by switching off the lamp and moving the probe. However, it is still possible to extrapolate the surface potential at the time the VUV is switched off using an exponential fit.

A first set of experiments have been performed with samples electrically connected to the chamber wall reference ground. A potential of $-4 \mathrm{~V}$ has been measured just after VUV irradiation. Two hypotheses have been proposed to explain this negative charging. The first hypothesis follows the grain super charging model [4] that assumes that negative charges on the top layer rear side overpasses the positive charging ion the top layer front side. Even though, the patch model has been corroborated by numerical simulation and by experiments at large scale [9], this hypothesis is difficult to assess experimentally at grain size. A patch structure does not necessarily means that the average potential would be negative. To clarify this point, a second hypothesis has been proposed. We indeed suspected a strong recollection by the dust layer of photoelectrons emitted by the copper plate. Low energy electrons emitted by the copper plate are indeed efficiently backscattered by the tank walls and reflected back inside the vacuum chamber, i.e. towards the dust layers [12]. If that repelled flux locally exceeded the flux emitted by the dust, that would explain the observed negative charging. Under these conditions, the setup would act as if the dust layer was under VUV and very large flux of primary environmental electron. To check this hypothesis, a second experiment has been conducted by applying a bias potential of $-45 \mathrm{~V}$ to the sample holder. The initial dust potential was thus -45 Volts. This technique is quite similar to the classical ones used for secondary electron emission measurement to reduce the backflow of repelled electrons where a positively biased hemispherical cup is placed above the sample [13]. The potential measured after VUV irradiation is $-33 \mathrm{~V}$ with respect to chamber ground. This corresponds to $\mathrm{a}+12 \mathrm{~V}$ differential potential with respect to the sample holder. That means that, in that configuration, dusts are positively charged in average.

This experimental campaign thus suggests that positive and negative average charging can alternatively be reached experimentally under VUV illumination pending on the electric field at the vicinity of the dust layer. This may be the 
reason why both positive and negative dust lofting was observed in the literature under VUV. The next section aims at providing more details on the positive charging experimental configuration under VUV using numerical simulations at macroscopic and microscopic scale.

\section{B. Numerical simulations}

Because of the large difference between individual grains size and the chamber dimensions, the electron transport and dust surface potentials are computed in two steps. First, a large scale simulation is performed using the geometry presented in Fig. 6. The DROP chamber is modelled by a $20 \mathrm{~cm}$-radius cupola. The circular target $1.5 \mathrm{~cm}$ in diameter surrounded by a rectangle of copper represents the dusts. The hole on the top represents the VUV lamp aperture. The mesh is refined at the dust layer location with mesh grid spacing of $0.5 \mathrm{~mm}$. It progressively increases up to $10 \mathrm{~mm}$ on the external part of the copper plate and up to $50 \mathrm{~mm}$ on the rest of the vacuum chamber. Similarly to the second experimental campaign, a bias potential of $-45 \mathrm{~V}$ has been imposed on the copper plate. All surface potentials are fixed except the dust one which is floating. In one hand, this simulation is used to compute the dust average surface potential. On the other hand, it is used to compute the electron fluxes coming in and out the dust layer vicinity to serve as inputs to a microscopic scale simulation.

The second simulation represents a dust pile geometry similar to what is shown in Section II. Grains are $25 \mu \mathrm{m}$-radius spheres and there are five layers, which results in a $0.2 \mathrm{~mm}$ thick sample. The mesh grid spacing is $2.5 \mu \mathrm{m}$ on dust grain and it progressively increases up to $125 \mu \mathrm{m}$ on the top boundary.

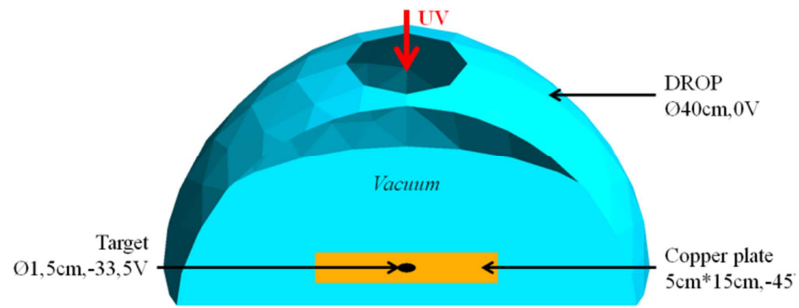

Fig. 6. 3D modelling of the DROP chamber simulated at macroscopic scale

\section{Results}

\section{1) Macroscopic simulation}

With the default SPIS material properties for dust, the surface potential is slightly more positive $(-30 \mathrm{~V})$ than during the experiments. The default value of the dust photoemission current density at (PEY) is $0.45 \mathrm{nA} / \mathrm{cm}^{2}$. The flux of photoelectrons emitted by a surface perpendicular to the VUV source is thus $9 \mathrm{nA} / \mathrm{cm} 2$, taking account of the VUV source amplification of 20 with respect to expected lunar conditions. With a PEY of $0.35 \mathrm{nA} / \mathrm{cm}^{2}$ instead, the average surface potential tends to the experimental value of $-33 \mathrm{~V}$. Fig . 7 presents a cross section of the plasma potential at $400 \mathrm{~s}$, i.e. after reaching equilibrium. Fig. 8 shows the photoelectron charge number density. At equilibrium, most of the photoelectrons emitted by the target plate are recollected by the target itself due to the presence if the potential barrier. One could obtain larger positive voltages by using larger dust deposit diameters. In addition, the photoelectron current emitted by the copper plate and recollected by the dust target surface is $I_{\text {recoll }}=3.3 \mathrm{nA}$. Fig. 9 presents the potential along the $\mathrm{Z}$ axis. A minimum potential of $-34.4 \mathrm{~V}$ is obtained at a distance of $3.5 \mathrm{~mm}$. This potential barrier is imposed by the $45 \mathrm{~V}$ copper plate and prevents the dust layer from charging more positive (or less negative) than $-33 \mathrm{~V}$ with respect to the tank walls.

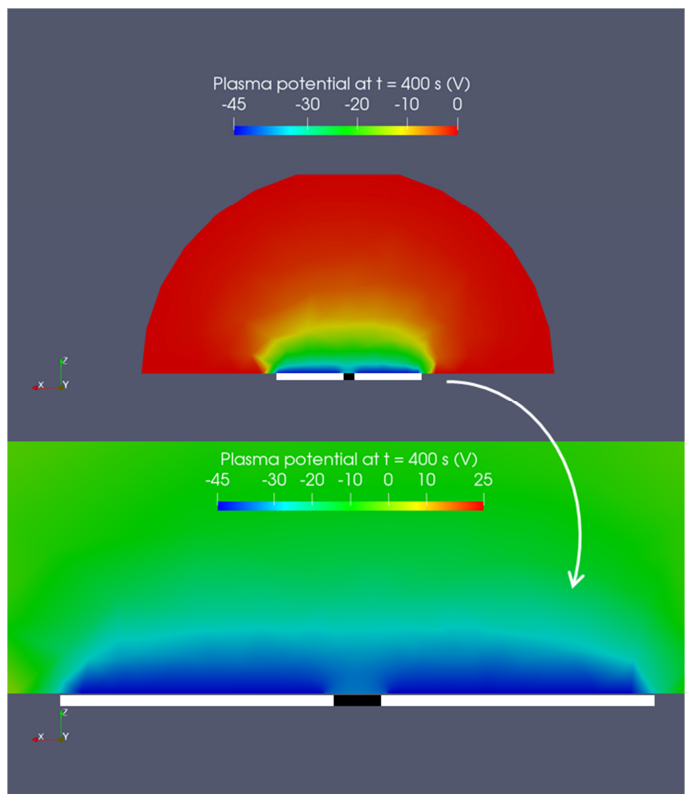

Fig. 7. Cross section of the computed potential inside the DROP chamber at equilibrium (top) and zoom around the dust layer (bottom). The copper plate and the dust layer are represented by the white and black rectangle, respectivelv.

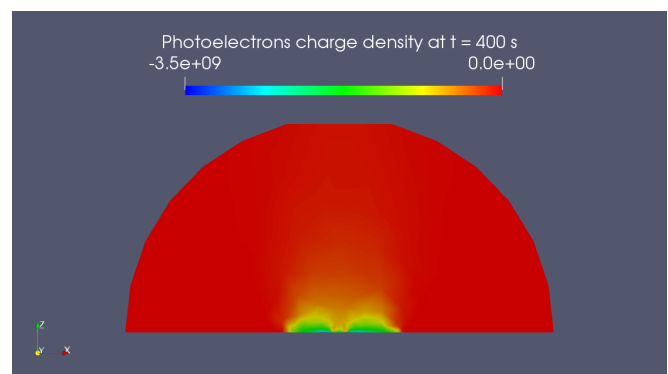

Fig. 8. Photoelectron charge number density $\left(\mathrm{m}^{-3}\right)$ at equilibrium. 


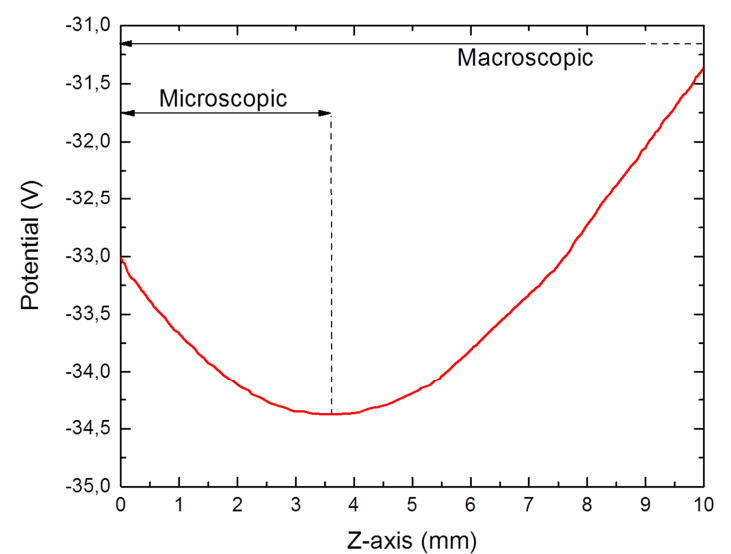

Fig. 9. Potential profile along the $\mathrm{Z}$ axis above the dust target.

\section{2) Microscopic simulations}

A quasi-1D domain is simulated, dimensioned using the location of the potential barrier created by the copper plate at approximately $3 \mathrm{~mm}$ from the dust layer. Above this barrier, all photoelectrons emitted by the dust layer are definitively lost, so that it can be modeled as an open boundary. A difference of potential of $-10.6 \mathrm{~V}$ is applied between the open boundary and the copper plate corresponding to the difference between the copper plate potential and the minimum barrier potential. For numerical reasons, all potentials are shifted by $34.4 \mathrm{~V}$ so the open boundary potential is $0 \mathrm{~V}$. This choice has not impact on the results since only potential gradients are important.

At this new scale, two electron populations are simulated: the photoelectrons emitted from the dust considering $\alpha=0^{\circ}$ and an isotropic Maxwellian population of electrons standing for the external photoelectron originating from the copper plate. Secondary electron emission is neglected here. The Maxwellian population is injected from the open boundary. Its density is determined in such a way that its thermal current density equals the current density of electrons coming from the copper plate obtained in the large scale simulation:

$$
\frac{I_{\text {recoll }}}{S}=\frac{1}{4} N q \sqrt{\frac{8 k_{B} T_{e}}{\pi m_{e}}},
$$

with $\mathrm{I}_{\text {recoll }}$ being the previously calculated current (in Ampere), $\mathrm{S}$ is the target surface (in square meter), $\mathrm{q}$ is the electron charge (in Coulomb), $\mathrm{k}_{\mathrm{B}}$ is the Boltzmann constant $\left(\mathrm{J}_{\mathrm{K}} \mathrm{K}^{-1}\right), \mathrm{T}_{\mathrm{e}}$ is the photoelectrons' temperature (in Kelvin) and $m_{e}$ their mass (in kilogram). The thermal electron temperature is fixed at $12 \mathrm{eV}$, which corresponds to the temperature of the electron emitted by the copper plus the potential difference between the copper plate and the potential barrier. Eventually, a density of $\mathrm{N}=1.95 \times 10^{8}$ particles $/ \mathrm{m}^{3}$ is found.

Fig. 10 presents the time evolution of the average potential over each layer. Fig. 11 shows a cross section of the surface potential after 450 seconds.

The potential on the first two layers is controlled by the external electron collection and photoelectron emission and recollection. First, photoelectrons are emitted and the potential goes up. Then, a fraction of them is collected as well as external electrons and the potential goes down until it reaches equilibrium within a few seconds. The differential potential between the top layer 1 and the copper plate is $+10 \mathrm{~V}$, in agreement with the experimental results. Grains that are not enlightened at all (three last in depth layers) do not emit any photoelectron, but they can collect electrons from the Maxwellian population. Thus, the potential on their surfaces decreases by a few volts, until equilibrium. The third layer gets a $-10 \mathrm{~V}$ potential with respect to the copper plate after about 10 minutes. The time scale to reach equilibrium is several hundreds of seconds. That means that experiments should be conducted over more than 10 minutes to get equilibrium state. The fourth and fifth layers collect very small amount of electrons.

The results show the same trends as in Section II suggesting that the experimental conditions produce results comparable to the expected lunar conditions. The chamber may be used to both simulate photoemission by dust and collection of ambient electrons. As for the simulation presented in Section II with $\alpha$ $=0^{\circ}$, the charge patch patterns are less pronounced. It would be interesting to test the effect of grazing incidence angle.

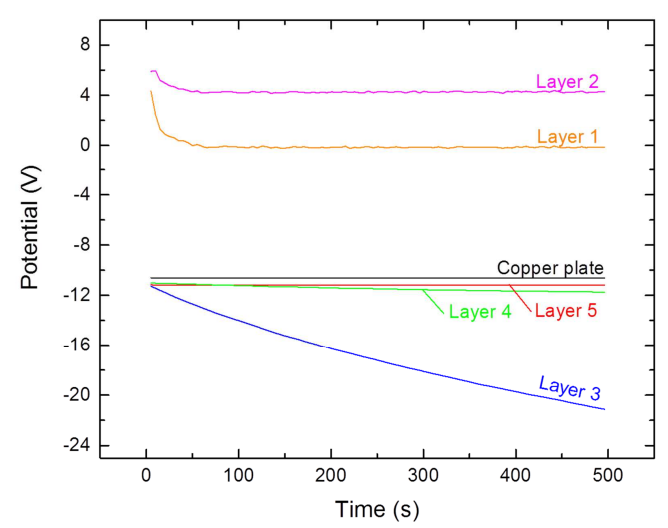

Fig. 10. Time evolution of the surface potentials of each dust layer

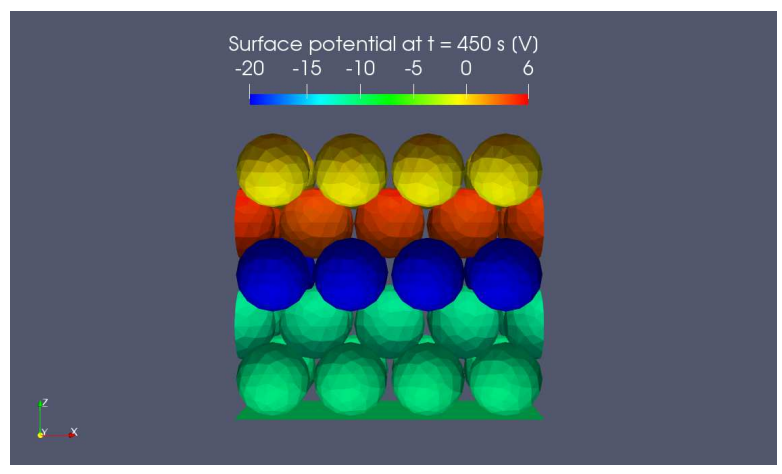

Fig. 11. Surface potentials cross section along the $\mathrm{Y}$ axis showing after $7 \mathrm{~min}$.

However, no charge conduction between layers is taken into account, which is not physically realistic. 


\section{3) Potential relaxation modelling}

We report here a study of charge relaxation after VUV illumination both experimentally and numerically. The time evolution of the surface potential have been measured at the center of the dust sample after 2 minutes of VUV irradiation is presented. In order to model numerically the potential relaxation, a current leakage is introduced between the layers by imposing an arbitrary resistance between two consecutive layers of $R=10^{17} \Omega$. The conducted current is computed by dividing the average potential difference between two layers by $R$. The value of $10^{17} \Omega$ is about ten times greater than the bulk internal resistance of each grain. The simulation duration is composed of 10 minutes initial charging under VUV followed by a relaxation of 110 minutes. Fig. 12 compares the potential evolution obtained both experimentally and numerically. Experimentally, surface potentials range from $+12 \mathrm{~V}$ to $0 \mathrm{~V}$ within $90 \mathrm{~min}$. Numerically, during the charging period, i.e. during the first 10 minutes, a behavior similar to the previous 3-layers simulations is observed. The first two layers reach an average potential of approximately +8 and $+5 \mathrm{~V}$. The third layer potential is decreasing due to electron collection. It reaches a minimum value at $-6 \mathrm{~V}$, which is quite superior to the $-25 \mathrm{~V}$ previously obtained for the same charging duration. The difference is due to the contact resistance simulated between layers which limit charge leakage from one layer to another. When the simulation reaches $10 \mathrm{~min}$ (VUV lamp is switch off), the two first layers show a potential decrease (the first decreasing more rapidly than the second one). Final simulated potentials reached are +4 and $+2 \mathrm{~V}$ after $120 \mathrm{~min}$. This difference potential is less important than the one reached from experimental results, indicating the simulated resistance contact value is too high. New estimation of the resistance contact is done using slope ratio between simulated and experimental potentials. In order to superpose both potential, it is found the simulated resistance contact must be about $10^{16} \Omega$.

Third layer shows a potential increase, seemingly providing charge to the fourth and fifth layer, as these two last ones show a potential decrease. Complex charge redistribution occurs and would need to be taken into during both charge and relaxation phases. However, supposing a relaxation time long enough, all potentials are expected to tend towards the copper plate potential.

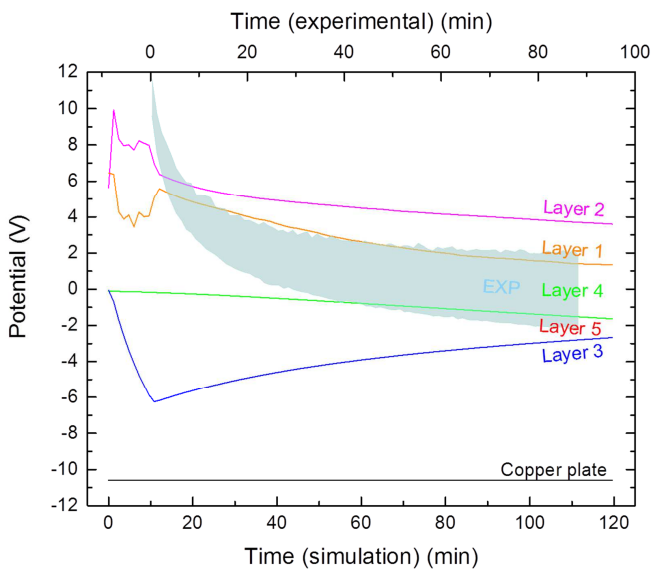

Fig. 12. Evolution of numerical surface potentials of layers 1 to 5 versus time (in continuous lines) and envelope of experimental potential relaxation measurements (in grey).

Fig. 113 presents a cross section of the five layers simulation at $120 \mathrm{~min}$. No more charge patches are observable, as resistance and conductivity have homogenized the potentials along the grains.

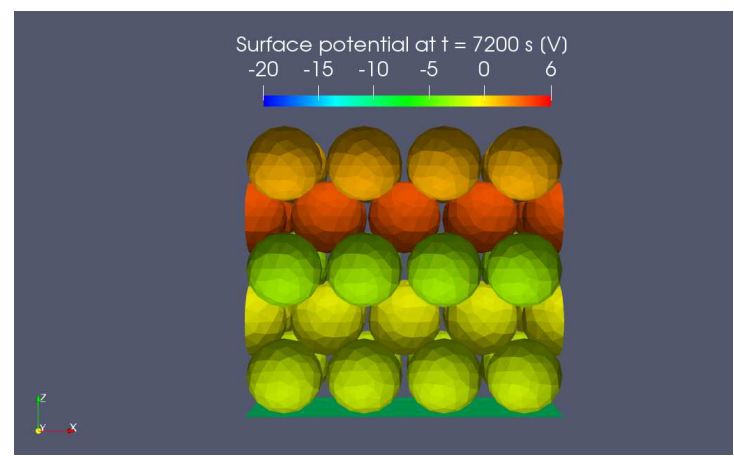

Fig. 13. Cross section along the $\mathrm{Y}$ axis showing surface potential at 120 min during potential relaxation.

\section{CONCLUSIONS}

This paper presented experimental and numerical evidence of complex charging patterns of dust submitted to lunar like environments. Average surface charging in sunlight may range from a few volts positive to a few volts negative pending on the soil geometrical configuration and electrical properties and on plasma conditions as well, including solar wind and sunlight incidence angle. We have shown that potential barriers are of prime importance to understand macroscopic charging behavior during ground testing. Extrapolating this observation to the lunar surface, one would expect for instance sunlit areas located at the border of negatively charged shaded surfaces to charge negatively due to the recollection of photoelectrons induced by potential barrier effects. This paper 
also corroborates literature models where dusts located on top of the lunar surface may exhibit positive and negative charge patches. We complement this model by showing the possibility of some dust buried quite deeply beneath the surface can get highly negative due to ambient electron collection. The charging time scale for these buried dusts is about a few hundreds of seconds while charging of the top layers dust is about a few seconds. That makes them more sensitive to electrical conduction mechanisms. The equilibrium potential of buried dust depends a lot on the soil bulk and surface conductivity as well as on the contact resistance between grains. The potential of dust located at the top surface is less sensitive but probably not totally insensitive to conduction processes, especially for smaller grains.

Electrostatic dust lofting has been reported in [4] on laboratory experiments even though no electrostatic force estimation could theoretically overcome adhesion and gravity. The observations made by the authors indicated aggregate lofting rather than single grain. The present paper, by showing that dust buried well beneath the top surface can get highly negatively charged, tends to corroborate what is suspected in [4]: electrical charge carriers can reach deeply inside aggregates and provide them sufficient charge level to get them mobilized by electrostatic lofting by an external electric field, that depends on large scale geometry, and/or by electrostatic repulsion with other dust and/or agglomerates, that is more related to small scale interaction.

Future work should include more conduction current measurements in order to assess charging attenuation by current leakage through dust and from grain to grain, pending on the lunar soil to be simulated. This is indeed a key parameter to understand charging patterns and their effect on dust mobilization and adhesion both at macroscopic and microscopic scales.

\section{ACKNOWLEDGEMENTS}

Authors would like to thank Serena Esposito from the Università degli Studi di Cassino and Francesca S. Freyria from the Instituto Politecnico di Torino for providing the SMA_A lunar dust simulant used in this paper.

\section{REFERENCES}

[1] Sandra A. Wagner, "The Apollo Experience Lessons Learned for COnstellation Lunar Dust Management." 17Jan-2008.

[2] D. R. Criswell, "Horizon-Glow and the Motion of Lunar Dust," in Photon and Particle Interactions with Surfaces in Space, vol. 37, R. J. L. Grard, Ed. Dordrecht: Springer Netherlands, 1973, pp. 545-556.

[3] M. Horanyi et al., "A permanent, asymmetric dust cloud around the Moon," Nature, vol. 522, no. 7556, pp. 324326, Jun. 2015.

[4] X. Wang, J. Schwan, H.-W. Hsu, E. Grün, and M. Horányi, "Dust charging and transport on airless planetary bodies: Electrostatic Dust Transport," Geophys. Res. Lett., vol. 43, no. 12, pp. 6103-6110, Jun. 2016.
[5] A. Champlain et al., "Lunar dust simulant charging and transport under UV irradiation in vacuum: Experiments and numerical modeling: ELECTROSTATIC

TRANSPORT OF LUNAR DUST," J. Geophys. Res. Space Phys., vol. 121, no. 1, pp. 103-116, Jan. 2016.

[6] Necmi Cihan Örger, J. Rodrigo Cordova-Alarcon, Kazuhiro Toyoda, and Mengu Cho, "Investigation of Electrostatic Transportation of Lunar Dust Grains due to Ambient Plasma Conditions." 2018.

[7] K. L. Mittal and R. Jaiswal, Eds., Particle Adhesion and Removal: Mittal/Particle. Hoboken, NJ, USA: John Wiley \& Sons, Inc., 2015.

[8] D. R. Criswell and B. R. De, "Intense localized photoelectric charging in the lunar sunset terminator region, 2. Supercharging at the progression of sunset," $J$. Geophys. Res., vol. 82, no. 7, pp. 1005-1007, Mar. 1977.

[9] M. I. Zimmerman et al., "Grain-scale supercharging and breakdown on airless regoliths: REGOLITH SUPERCHARGING AND BREAKDOWN," J. Geophys. Res. Planets, vol. 121, no. 10, pp. 2150-2165, Oct. 2016.

[10] Jean-Charles Matéo-Vélez, Bernard Dirassen, Mohamed Belhaj, and Jean-Pierre Chardon, "Development of a predictive discharge numerical model on solar panels Test Report," May 2010.

[11] F. Freyria et al., "Simulated Moon agglutinates obtained from zeolite precursor by means of a low-cost and scalable synthesis method," Under review, 2019.

[12] R. Cimino, "Can Low Energy Electrons Affect High Energy Physics Accelerators?," SLAC-PUB-10350, 826848, Apr. 2004.

[13] M. Belhaj, T. Tondu, V. Inguimbert, P. Barroy, F. Silva, and A. Gicquel, "The effects of incident electron current density and temperature on the total electron emission yield of polycrystalline CVD diamond," J. Phys. Appl. Phys., vol. 43, no. 13, p. 135303, Apr. 2010. 